\title{
GROUP THEORY BASED AT ANY POINT
}

\author{
M. A. ALBAR and S. A. HUQ
}

(Received 22 September 1997 and in revised form 20 July 1998)

\begin{abstract}
If $(G, \cdot)$ is a group with identity $e$, we call $G$, the group based at $e$. In this paper, we aim to release the present day group theory which is based at $e$, by replacing $e$ by an arbitrary element of the group.
\end{abstract}

2000 Mathematics Subject Classification. 20A05, $20 \mathrm{~F} 12$.

1. Introduction. If $(G, \cdot)$ is a group with the identity $e$, we call $G$ a group based at $e$. We can change this base to any other element $a \in G$, by defining a new sandwich multiplication, $\stackrel{*}{a}$, called the multiplication based at $a$, as

$$
p \stackrel{*}{a} q=p a^{-1} q ; \quad p, q \in G
$$

making $(G, \stackrel{*}{a})$, a group now based at $a$. It was previously known $[1,2]$ that all these structures at different based points are isomorphic.

Thus the group theoretic concepts, presently available at $(G, \cdot)$ based at $e$ are rather special cases of the more general concepts based at an arbitrary point $a \in G$, called now the concepts based at $a$. The idea here is parallel to that in differential geometry, where one chooses a point on a smooth manifold and then studies differential geometric concepts like that of, say, a "tangent bundle" at that point [3].

If a concept based at $a$ holds for all $a \in G$, we call this an everywhere concept in $G$.

In this paper, we briefly underline these ideas. Our study arose while looking at concepts for pregroups mentioned in our previous work [2]. We use the notation of [2], by replacing $\hat{a}$, by $\stackrel{*}{a}$ for simplification.

Throughout, we denote $(G, \cdot)$ for the group based at $e$, and $a$ denotes an arbitrary fixed element of $G$.

\section{Based subgroups}

DEFINITION 2.1. A nonempty subset $H$ of a group $G$ is called an $a$-based subgroup of $G$ if

$$
p, q \in H \text { implies } p q^{-1} a \in H \text {. }
$$

We use the notation $H \leq_{a} G$.

The usual notion of a subgroup in the common group theoretic sense is that of an $e$-based one for the identity element $e$ of $G$. By definition if $H$ is an $a$-based subgroup, then $a \in H$. 
It is quite clear that for two distinct $a, b \in G$, an $a$-based subgroup need not be $b$-based, as can be seen from the following example.

EXAMPLE 2.2. The positive rationals, with respect to multiplication, denoted by $\left(\mathbb{Q}^{+}, \cdot\right)$ is an identity based subgroup of $\left(\mathbb{R}^{+}, \cdot\right)$ of all positive reals, but not an $r$-based subgroup of an irrational $r \in \mathbb{R}^{+}$.

Our next proposition determines when a nonempty subset $H$ of a group $(G, \cdot)$ based at $e$ is an $a$-based subgroup for $a \in G$ and is an adapted version of a result due to Certaine [1].

THEOREM 2.3. A nonempty subset $H$ of a group $G$ is an a-based subgroup of $G$ if $H=U a$ or aU for the subgroup $U=H a^{-1}$ (or $a^{-1} H$ ). Conversely, if $H=U a$ (or $a U$ ) for any subgroup $U$ of $G$, then $H$ is an a-based subgroup of $G$.

Proof. Suppose that $H$ is an $a$-based subgroup. Then for $p, q \in H ; p q^{-1} a \in H$, or in other words,

$$
p, q \in H \quad \text { implies } p q^{-1} \in H a^{-1} \text {. }
$$

This is precisely the condition that

$$
U=H a^{-1} \text { is a subgroup of } G \text {. }
$$

For if $u_{1}, u_{2} \in U$, then $u_{1}=h a^{-1}$ and $u_{2}=h^{\prime} a^{-1} ; h, h^{\prime} \in H$. Then $u_{1} u_{2}^{-1}=$ $h a^{-1} a h^{\prime-1}=h h^{\prime-1} \in H a^{-1}$ by condition (2.2). So $U=H a^{-1}$ is a subgroup of $G$ and $U a=H$.

Conversely, suppose that $H=U a$ for a subgroup $U$ of $G$, then for $p, q \in H, p=u a$, $g=u^{\prime} a$, thus

$$
p q^{-1} a=u a a^{-1} u^{\prime-1} a=u u^{\prime-1} \in U a=H .
$$

Thus $H$ is an $a$-based subgroup.

The proof when $H$ is a left $a$-coset is similar. Thus the $a$-based subgroups are nothing but $a$-cosets (left and right).

The next result is rather simple.

Proposition 2.4. For two elements $a, b$ and a subgroup $H$ of $G, H$ is a-based implies $H$ is $b$-based, if and only if $a^{-1} b \in H$, that is, $b H=a H$ (or $a b^{-1} \in H$, i.e., $H a=H b$ ). That is, if $a, b \in G$ and $H \leq_{a} G$, then $H \leq_{a} G$ if and only if $a^{-1} b \in H$.

Thus for two elements $a$ and $b$ belonging to a subgroup $H, H$ is $a$-based always implies $H$ is $b$-based. We notice that any subgroup $H$ is an everywhere subgroup if and only if $H=G$, itself, and the group consisting of a single element is only that element based subgroup that is a nowhere subgroup.

\section{Based abelianness}

DEFINITION 3.1. A subgroup $H$ of $(G, \stackrel{*}{a})$ is called $a$-based abelian, if for $p, q \in H$,

$$
p a^{-1} q=q a^{-1} p
$$


From the definition it is clear that $H$ is $a$-based abelian if

$$
p a^{-1} q=\left(p^{-1} a q^{-1}\right)^{-1}
$$

that is,

$$
p^{-1} a q^{-1} p a^{-1} q=e \quad \text { or } \quad a p^{-1} a q^{-1} p a^{-1} q=a .
$$

Proposition 3.2. For a subgroup $H$ of the group $(G, \cdot)$ based at e, and two distinct elements $a, b \in H$, if $H$ is $a$-based abelian then $H$ is $b$-based abelian also.

Proof. This follows from [2, Theorem 3.2], which asserts $(H, \stackrel{*}{a}) \simeq(H, \stackrel{*}{b})$.

COROLlary 3.3. A subgroup $H$ is abelian if and only if it is a-based, for one $a \in H$.

Proof. If $H$ is $a$-based abelian, then for $p, q \in H, p a^{-1} q=q a^{-1} p$, so $H$ is $a$-based abelian.

Conversely, if $H$ is $a$-based abelian, then $H$ is $e$-based abelian also, for the identity element $e$ of $G$; that is, $H$ is abelian.

Thus an $a$-based abelian group $G$, for $a \in G$, is exactly an abelian group in the common group theoretic sense.

Next we examine this concept for an $a$-based subgroup $H$ of $(G, \cdot)$.

Proposition 3.4. An a-based subgroup $\mathrm{H}$ is a-based abelian if and only if $\mathrm{H}=U \mathrm{Ua}$ (or $a U$ ) for an abelian subgroup $U$ of $G$.

Proof. By Theorem 2.3, $H$ is an $a$-based subgroup if and only if $H$ has the required stated form. Now, for $h_{1}, h_{2} \in H, h_{1}=u_{1} a$ and $h_{2}=u_{2} a$. So $h_{1} a^{-1} h_{2}=h_{2} a^{-1} h_{1}$ gives

$$
u_{1} a a^{-1} u_{2} a=u_{2} a a^{-1} u_{1} a,
$$

that is, $u_{1} u_{2}=u_{2} u_{1}$. So $U$ is abelian.

Conversely $U$ is abelian, gives $u_{1} u_{2} a=u_{2} u_{1} a$ for an $a \in G$ which gives $h_{1} a^{-1} h_{2}=$ $h_{2} a^{-1} h_{1}$ for $h_{1}=u_{1} a$ and $h_{2}=u_{2} a$. Thus $H=U a$ is $a$-based abelian.

\section{Based normality}

DEFINITION 4.1. A subgroup $H$ of $G$ is called $a$-based normal, denoted by $H \underset{a}{\triangleleft} G$, if for any $x \in G$,

$$
a x^{-1} h a^{-1} x \in H \quad \forall h \in H .
$$

From the definition, it is clear that

(a) a subgroup $H$ is $a$-based normal, if $a(x H) a^{-1} \subseteq H x$, that is, the inner automorphism by $a$ of $G$, translates a left coset into a right coset,

(b) a subgroup $H$ is everywhere normal if $\mathrm{Ha}^{-1} x \subseteq x a^{-1} H$ for every pair of elements $a, x \in G$, that is, $H a b \subseteq b a H$ for all $a, b \in G$.

REMARK 4.2. (i) If $G$ is abelian, all subgroups are everywhere normal. Thus it follows from the example in Section 2 that an $a$-based normal subgroup need not be an $a$ based subgroup (for an irrational $a$ in that example), as $a$ need not belong to $H$. Conversely, every element is that element based (trivial) normal subgroup in the group 
based at that element. Thus, it becomes more natural to consider an a-based subgroup which is a-based normal as well. When we wish to indicate this, we write $H \underbrace{a}_{a} G$, meaning $H$ is an a-based subgroup for which condition (i) holds.

(ii) If every pair of elements $(a, x)$ of the group $G$ is inverse commuting (i.e., the inverse of one commutes with the other), then every normal subgroup of $G$ is everywhere normal.

One now asks when is a subgroup $a$-based normal for an $a \in G$ ? The answer is as follows.

THEOREM 4.3. If $G$ is a group, $a \in G$ and $H$ a subgroup of $G$ then $H \underset{a}{\triangleleft} G$, if and only if

(i) $H \triangleleft G$,

(ii) $\left[a^{-1}, G\right] \subseteq H$, where $\left[a^{-1}, G\right] \subseteq H$ means $\left[a^{-1}, x\right] \in H$ for every $x \in G$.

Proof. Let $H \underset{a}{\triangleleft} G$, then for all $x \in G$ and all $h \in H, a x^{-1} h a^{-1} x \in H$, that is, $a x^{-1} h x a^{-1} a^{-1} x \in H$ or $\left(x a^{-1}\right)^{-1} h\left(x a^{-1}\right)\left[a^{-1}, x\right] \in H$. Choosing $h=e$, this gives for all $x \in G,\left[a^{-1}, x\right] \in H$. This is (ii).

Conversely, for all $x \in G, h \in H,\left(x a^{-1}\right)^{-1} h\left(x a^{-1}\right) \in H$. Next let $y \in G$, then for all $h \in H$,

$$
y^{-1} h y=\left(y a a^{-1}\right)^{-1} h\left(y a a^{-1}\right) \in H \quad \text { and therefore } H \triangleleft G .
$$

Conversely, let $H$ satisfy (i) and (ii). Then by (i) for all $x \in G$ and all $h \in H$,

$$
\left(x a^{-1}\right)^{-1} h\left(x a^{-1}\right) \in H,
$$

and by (ii)

$$
\left[a^{-1}, x\right] \in H
$$

thus $\left(x a^{-1}\right)^{-1} h\left(x a^{-1}\right)\left[a^{-1}, x\right] \in H$. That is, $a x^{-1} h a^{-1} x \in H$ and so $H \underset{a}{\triangleleft} G$.

COROLLARY 4.4. Let $G$ be a subgroup, $a \in G$ and $H$ a subgroup of $G$, then $H \unlhd a$, if and only if

(i) $H \triangleleft G$,

(ii) the coset $\mathrm{Ha}$ is in the center of $\mathrm{G} / \mathrm{H}$.

It follows immediately that a normal subgroup $H$ of $G$ is everywhere normal if and only if the derived group $G^{\prime}=[G, G] \subseteq H$, that is, if and only if $G / H$ is abelian.

As we noticed, the normal closure $\overline{\left[a^{-1}, G\right]}$ of $\left[a^{-1}, G\right]$ is an $a$-based normal subgroup of $G$. This is called the a-derived subgroup of $G$. In fact this is the smallest $a$-based normal subgroup of $G$.

Notice that the derived group $G^{\prime} \triangleleft G$ and $\left[a^{-1}, G\right] \subseteq G^{\prime}$; in fact $\left[a^{-1}, G\right]^{t} \subseteq G^{\prime}$ for $t \in G$.

DEFinITION 4.5. A group $G$ is called $a$-solvable (of class $k$ ), for an $a \in G$, if $\left[a^{-1}, G^{k}\right]$ $\subseteq G^{k+1}=1$.

Obviously, every subgroup of an a-solvable subgroup is a-solvable, and every solvable group is everywhere solvable. 
Returning again to based normality, we notice that if in Definition 4.1, we assume that (i) holds for only a fixed $x \in G$, we call $H \underset{a}{\triangleleft} G$, relative to the element $x \in G$. With this convention we conclude that if $H \underset{a}{\triangleleft} G$, relative to $b \in G$, then $H_{b^{-1}}^{\triangleleft} G$, relative to $a$.

THEOREM 4.6. For any $a \in G$,

(i) $H \unlhd \underset{a}{\triangleleft}, H \triangleleft G$ implies $H K \underset{a}{\triangleleft} G$,

(ii) $A \underset{a}{\triangleleft} G, B \underset{a}{\triangleleft} G$ implies $A \cap B \underset{a}{\triangleleft} G$, (this remains valid when $a$ is replaced by $\underbrace{a}$ ),

(iii) if further $a \in H$, then $K \underset{a}{\triangleleft} G, H$ a-subgroup of $G$ implies $H \cap K \underset{a}{\triangleleft} H$.

Proof. Since the proofs are routine verification as in common group theory, we exhibit typically the proof of (i).

To see (i), notice $H K$ is a normal subgroup, since $H$ and $K$ are, and

$$
\left[a^{-1}, G\right] \subseteq H \subseteq H K
$$

Finally, we exhibit a characterization of $a$-based subgroups which are $a$-based normal as well. Our result is an adapted version of our previous result [2, Theorem 8.1] for this case, though the proof is completely new.

THEOREM 4.7. An a-based subgroup $H$ is a-based normal $\left(H_{\underbrace{a}_{a}}^{G}\right)$ if and only if $H=U a(=a U)$ where $U \triangleleft G$.

Proof. Notice that $H$ is an $a$-based subgroup if and only if $H=U a$ (or $a U$ ) where $U$ is a subgroup by Theorem 2.3 .

Now if $H$ is an $a$-based normal subgroup, then for any $x \in G$,

$$
a x^{-1} H a^{-1} x \subseteq H,
$$

that is, $a x^{-1} U x \subseteq H=U a$, thus $a x^{-1} U x a^{-1} \subseteq U$ or in other words

$$
\left(x a^{-1}\right)^{-1} U x a^{-1} \subseteq U .
$$

Showing $U \triangleleft G$, as in the proof of Theorem 4.3, since $U a=a U$, the result is true for $a U$ also.

Conversely, if $H=U a$, where $U \triangleleft G$, then

$$
\begin{aligned}
a x^{-1} H a^{-1} x & =a x^{-1} U a a^{-1} x \quad \text { for } u \in U \\
& =a x^{-1} u x \\
& =a u^{\prime} \quad \text { for } u^{\prime}=x^{-1} u x \in U \\
& \in a U=U a=H .
\end{aligned}
$$

5. Based direct product. Let $\left(A_{i}\right)_{i \in I}$ be a family of groups with respect to multiplication. Let $k_{i} \in A_{i}$, then we define the based direct product $\prod_{\left(k_{i}\right)_{i \in I}} A_{i}$, as follows.

For $\left(a_{i}\right)_{i \in I},\left(b_{i}\right)_{i \in I}$, define

$$
\left(a_{i}\right)_{i \in I}\left(k_{i}^{*}\right)_{i \in I}\left(b_{i}\right)_{i \in I}, \quad \text { by }\left(a_{i} k_{i} b_{i}\right)_{i \in I^{*}}
$$


This makes $\pi A_{i}$ a direct product based at $\left(k_{i}\right)_{i \in I}$ usually denoted by $\prod_{\left(k_{i}\right)_{i \in I}} A_{i}$. Notice that, the inverse $\left[\left(a_{i}\right)_{i \in I}\right]^{-1}=\left[\begin{array}{c}a_{*}^{-1} \\ i k_{i}\end{array}\right]_{i \in I}$, where $a_{i k_{i}}^{-1}$ denote the inverse of $a_{i}$ with respect to the multiplication based at $k_{i}$ [2].

With this definition of product, usual group theoretic concepts could be extended to hold at an arbitrary point. A simplest theorem of this nature is as follows.

THEOREM 5.1. $A_{i} \underbrace{\triangleleft}_{a_{i}} B_{i}$ implies $\prod_{\left(a_{i}\right)_{i \in I}} A_{i} \underbrace{a_{\left(a_{i}\right)_{i \in I}}}_{\underbrace{}_{i})_{i \in I}^{\triangleleft}} B_{i}$, where $a_{i} \in A_{i}$ for each $i$.

\section{Homomorphisms}

DeFinition 6.1. Let $G$ and $H$ be two groups; $a \in G, b \in H$. We call a mapping $\phi: G \rightarrow H$, a homomorphism, if for $g_{1}, g_{2} \in G$,

$$
\phi\left(g_{1} \stackrel{*}{a} g_{2}\right)=\phi\left(g_{1}\right) \stackrel{*}{b} \phi\left(g_{2}\right)
$$

that is,

$$
\phi\left(g_{1} a^{-1} g_{2}\right)=\phi\left(g_{1}\right) b^{-1} \phi^{\prime}\left(g_{2}\right) .
$$

Choosing $g_{1}=g_{2}=a$, we have $b=\phi(a)$, that is, the homomorphism preserves the base point.

Proposition 6.2. If $\phi: G \rightarrow H$ is a homomorphism, then

(i) $K \underbrace{\triangleleft}_{a} G$ implies $K \phi \underbrace{\triangleleft}_{\sim} G \phi$,

(ii) $K \underbrace{\triangleleft}_{b} H$ implies $K^{-1} \phi \underbrace{\triangleleft}_{a} G$, can then be formulated.

7. Based commutators. For two elements $p, q$ of $G$, we define for a fixed element $a \in G$, an $a$-based commutator of $p$, and $q$ by

$$
[p, q]_{a}=a p^{-1} a q^{-1} p a^{-1} q .
$$

Thus $[p, q]_{e}$ for the identity $e$, is the usual commutator.

From the definition, it is immediate

$$
[p, q]_{a}^{-1}=q^{-1} a p^{-1} q a^{-1} p a^{-1} .
$$

Thus

$$
a[p, q]_{a}^{-1} a=[q, p]_{a}
$$

Also,

$$
[p, p]_{a}=a
$$

Notice

$$
[p, q]_{a}=\left[\left(p a^{-1}\right),\left(q a^{-1}\right)\right]_{e} a .
$$

Besides these, we record the general commutator identities as follows.

THEOREM 7.1 (see [2]). (i) $\left[p, q a^{-1} r\right]_{a}=[p, r]_{a} a^{-1}\left([p, q]_{a}\right)^{r}$.

(ii) $\left[p a^{-1} q, r\right]_{a}=\left([p, r]_{a}\right)^{q} a^{-1}[q, r]_{a}$, where $x^{p}=p_{a}^{-1} a^{-1} x a^{-1} p$, where $p_{a}^{-1}$ is the a-based inverse of $p$. 
Proof. As usual, we exhibit the proof of (ii). The left-hand side of (ii)

$$
\begin{aligned}
{\left[p a^{-1} q, r\right]_{a} } & =a\left(p a^{-1} q\right)^{-1} a r^{-1}\left(p a^{-1} q\right) a^{-1} r \\
& =a q^{-1} a p^{-1} a r^{-1} p a^{-1} q a^{-1} r .
\end{aligned}
$$

Also the right-hand side

$$
\begin{aligned}
\mathfrak{q}_{a}^{-1} a^{-1}[p, r]_{a} a^{-1} q a^{-1}[q, r]_{a} & =a q^{-1} a p^{-1} a r^{-1} p a^{-1} r a^{-1} q a^{-1} a q^{-1} a r^{-1} q a^{-1} r \\
& =a q^{-1} a p^{-1} a r^{-1} p a^{-1} q a^{-1} r .
\end{aligned}
$$

This completes the proof.

Obviously, if $[p, q]_{a}=a$ for any $p, q$ and $a \in G$, then $G$ is $a$-based abelian therefore abelian by Corollary 3.3 of Proposition 3.2.

It is now quite clear how one generalizes various other concepts. For example, in $G$, two sets $S$ and $S^{\prime}$ are $a$-based conjugate, for an $a \in G$, if there exists an $x \in G$, such that $a x^{-1} S a^{-1} x=S^{\prime}$. If this is true for every $a \in G$, we obtain an everywhere conjugate pair of sets.

The study of $\underbrace{a}$-based normalizer, $\underbrace{a}$-based centralizer, and so forth, follows the direction of $\underbrace{a}$-based normal subgroups ( $a$-based subgroups which are $a$-based normal) and their everywhere counterparts are then a matter of routine. We leave these as exercises.

REMARK 7.2. (a) Let $F$ be a free group on a set $S$ of symbols. Adjoin a symbol $a$ to $S$ to get $S \cup\{a\}$. The free group on $S \cup\{a\}$ could be considered as a free group on $S$ based at $a$, denoted by $F_{a}$ and any two such $F_{a}$ and $F_{b}$ are isomorphic for different symbols $a, b$.

If $a, b \in S$, there is no difference between $F_{a}, F_{b}$, or $F$. Thus a free group on a set $S$ could be considered freely based at every point of $S$, that is everywhere free on $S$.

(b) Though we have studied $(G, \stackrel{*}{a})$ in terms of the usual $(G, \cdot)=(G, \stackrel{*}{e})$ for the identity $e$, with which we are familiar, it was quite possible to study the situation in terms of $(G, \stackrel{*}{b})$ based at $b$.

For example a version of Theorem 4.3 states that "An a-based subgroup $H_{a} \underset{a}{\triangleleft} G_{a}^{*}$, if and only if $H_{a} \underset{a}{\triangleleft} G_{a}$ and $\left[b_{a}^{-1}, G_{a}^{*}\right]_{a} \subseteq H_{a}$."

We are content to leave our reader to these entertaining exercises again. In a subsequent paper, we look forward to use our results in differential geometry to study how a principal fiber bundle at any point in a group could be located through that at the identity. Behavior of topological groups under change of base points appears to be an interesting area of study. The group theory where any element of the group could play the role of the identity is of interest to computer science as well.

One notices that our situation generalizes suitably to other algebraic structures.

Acknowledgement. The first author thanks King Fahd University of Petroleum and Minerals, Dhahran, Saudi Arabia, for the support he gets for conducting research. 


\section{REFERENCES}

[1] J. Certaine, The ternary operation $(a b c)=a b^{-1} c$ of a group, Bull. Amer. Math. Soc. 49 (1943), 869-877. MR 5,227f. Zbl 061.02305.

[2] S. A. Huq, Some aspects of pregroups, Arch. Math. (Basel) 47 (1986), no. 6, 522-528. MR 87m:20191. Zbl 594.20073.

[3] J. A. Lees, Notes on Bundle Theory, Lecture Notes in Series, no. 42, Matematisk Institut, Aarhus Universitet, Aarhus, 1974. MR 50\#11235. Zbl 304.55014.

M. A. Albar ANd S. A. HuQ: Department of MAthematical Sciences, King Fahd UniverSity of PETROleum AND Minerals, DHAhran 31261, SAUdi ARABia 


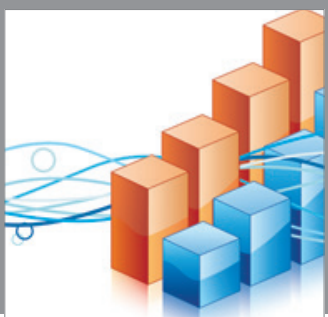

Advances in

Operations Research

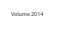

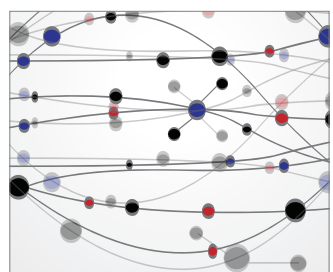

\section{The Scientific} World Journal
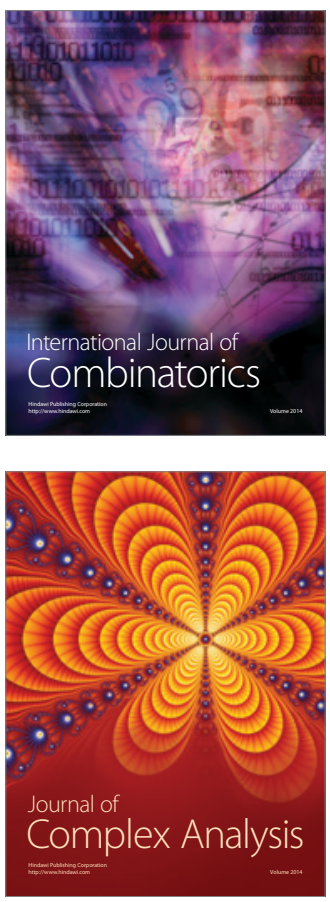

International Journal of

Mathematics and

Mathematical

Sciences
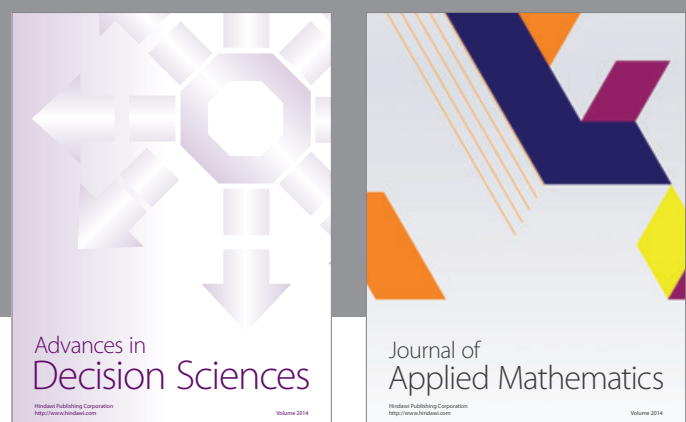

Journal of

Applied Mathematics
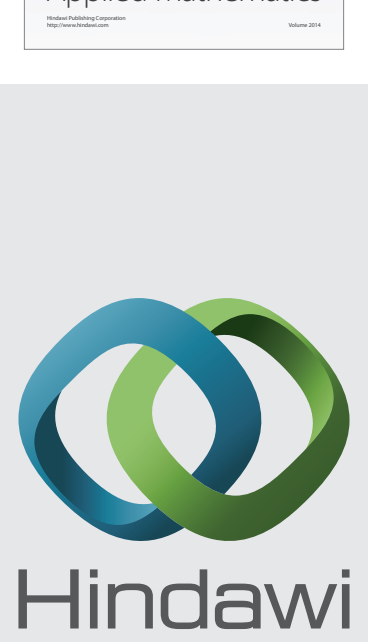

Submit your manuscripts at http://www.hindawi.com
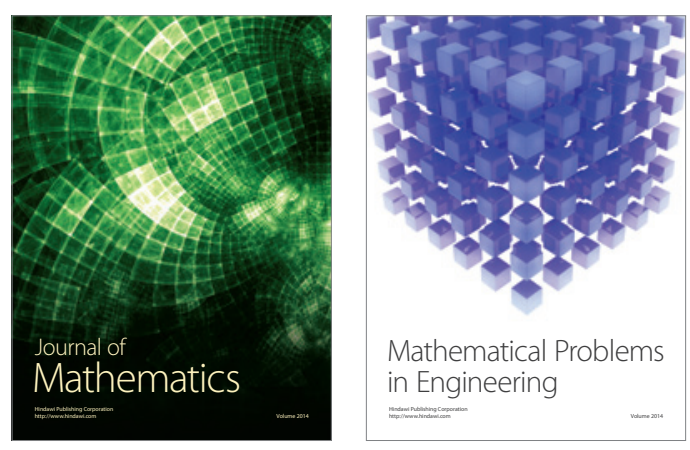

Mathematical Problems in Engineering
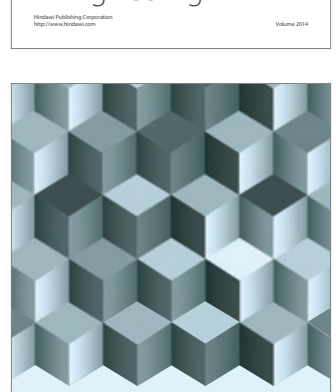

Journal of

Function Spaces
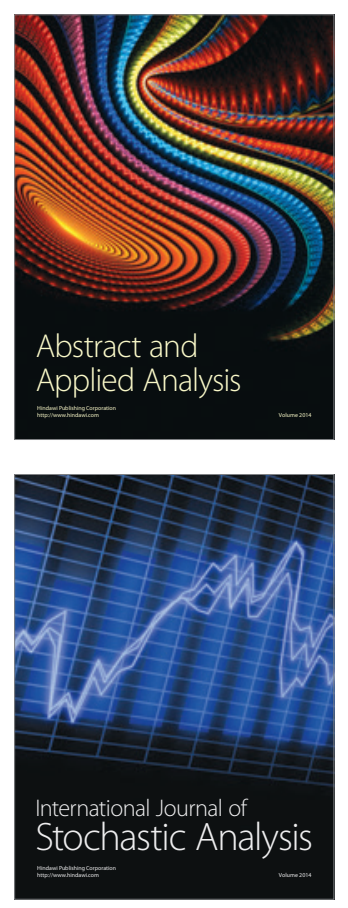

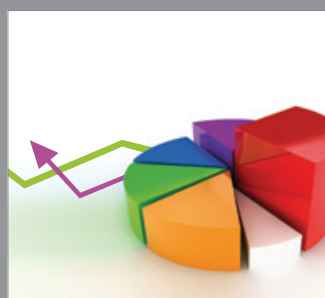

ournal of

Probability and Statistics

Promensencen
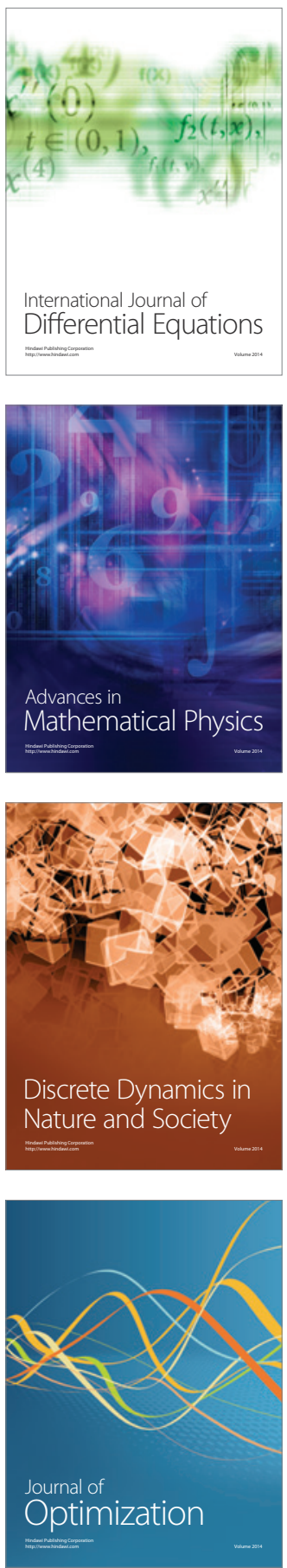